\title{
Predictors of recurrence and disease-free survival in patients with completely resected esophageal carcinoma
}

\author{
Paul C. Lee, MD, ${ }^{\text {a }}$ Farooq M. Mirza, MD, ${ }^{a}$ Jeffrey L. Port, MD, ${ }^{a}$ Brendon M. Stiles, MD, ${ }^{a}$ \\ Subroto Paul, MD, ${ }^{\mathrm{a}}$ Paul Christos, DrPH, ${ }^{\mathrm{b}}$ and Nasser K. Altorki, $\mathrm{MD}^{\mathrm{a}}$
}

\begin{abstract}
Objective: The goal of this study was to analyze factors predictive of recurrence and disease-free survival in patients with completely resected esophageal carcinoma.
\end{abstract}

\begin{abstract}
Methods: We conducted a retrospective review of a prospective database to identify patients with completely resected esophageal carcinoma. Medical records were reviewed. Recurrence rates, time to recurrence, and disease-free survival were analyzed. The Kaplan-Meier method was used for time to event estimation, and multivariate Cox regression models were constructed to analyze factors thought to be significant in determining both freedom from recurrence and disease-free survival.
\end{abstract}

Results: From 1988 to 2009, 465 of 500 patients underwent complete resection for esophageal carcinoma. Median follow-up for living patients was 49 months; 197 patients (42.4\%) had recurrence, leading to 175 patients dying of cancer and 22 patients living with recurrent disease. Multivariate regression adjusted for $P$ stage identified the following variables as independent predictors of freedom from recurrence: performance status greater than 0 (hazard ratio [HR], 1.84; 95 confidence interval [CI], 1.35-2.49]; $P<.001$ ), poor differentiation (HR, 1.50; CI, 1.12-2.01; $P=.006$ ), induction therapy (HR, 1.65; CI, 1.21-2.25]; $P=.002)$, en bloc resection (HR, 0.61; CI, 0.43-0.88; $P=.007$ ), and advanced pathologic stages (II/III/IV) (HR, 5.46; CI, 3.05-9.78; $P<.001$ ). Independent predictors of disease-free survival adjusted for $P$ stage were performance status greater than 0 (HR, 1.73; CI, 1.34-2.23; $P<.001$ ), en bloc resection (HR, 0.63; CI, 0.47-0.84; $P=.002$ ), induction therapy (HR, 1.34; CI, 1.02-1.76; $P=.033$ ), and advanced pathologic stages (II/III/IV) (HR, 3.16; CI, 2.15-4.65; $P<.001)$.

Conclusions: For patients with completely resected esophageal cancer, independent predictors of improved freedom from recurrence and disease-free survival include good performance status, en bloc resection, and early pathologic stage. (J Thorac Cardiovasc Surg 2011;141:1196-206)

Earn CME credits at

http://cme.ctsnetjournals.org

The diagnosis of esophageal carcinoma carries a dismal prognosis for the majority of patients, with more than $95 \%$ of patients succumbing to their disease. Among the subset of patients resected with curative intent (R-0 resection), the 5 -year survival of patients after transthoracic esophagec-

From the Division of Thoracic Surgery, ${ }^{\mathrm{a}}$ Department of Cardiothoracic Surgery, and Division of Biostatistics and Epidemiology, ${ }^{\mathrm{b}}$ Department of Public Health, New York Presbyterian Hospital, Weill Cornell Medical College, New York, NY.

Dr Paul Christos was partially supported by Grant UL1-RR024996 from the Clinical Translational Science Center.

Disclosures: Authors have nothing to disclose with regard to commercial support.

Read at the 90th Annual Meeting of The American Association for Thoracic surgery, Toronto, Ontario, Canada, May 1-5, 2010.

Received for publication May 1, 2010; revisions received Dec 10, 2010; accepted for publication Jan 7, 2011; available ahead of print March 14, 2011.

Address for reprints: Paul C. Lee, MD, Department of Cardiothoracic Surgery, Suite M404, Weill Medical College of Cornell University, 525 East 68th Street, New York, NY 10021 (E-mail: pc19001@med.cornell.edu).

0022-5223/\$36.00

Copyright (C) 2011 by The American Association for Thoracic Surgery doi: $10.1016 /$ j.jtcvs.2011.01.053 tomy or transhiatal esophagectomy rarely exceeds $30 \% .^{1-4}$ The primary reason for the poor results is that metastatic disease develops in the majority of patients, suggesting that the disease may already have disseminated at the time of diagnosis. Although undoubtedly this is the case in some patients, a careful analysis of the patterns of failure after various local therapies delivered with curative intent suggests inadequate locoregional control. For example, the locoregional failure rate after definitive chemoradiation is in the $30 \%$ to $40 \%$ range. ${ }^{5}$ Similarly, local failure rates after surgical resection have been reported to be in the $30 \%$ to $40 \%$ range. $^{6-9}$ Factors affecting postoperative recurrence and disease-free survival (DFS) are often unclear. The goal of this study was to review our surgical experience in patients with completely resected esophageal carcinoma and to determine predictors of recurrence and long-term DFS.

\section{PATIENTS AND METHODS Patients}

We conducted an institutional review board-approved retrospective review of a prospectively maintained database to identify patients who underwent esophagectomy for invasive carcinoma of the esophagus and gastroesophageal junction between November 1987 and December 2009. Patients with high-grade dysplasia were excluded. Hospital and office 

Abbreviations and Acronyms
$\mathrm{CI}=$ confidence interval
$\mathrm{CT}=$ computed tomography
$\mathrm{DFS}=$ disease-free survival
$\mathrm{FFR}=$ freedom from recurrence
$\mathrm{HR}=$ hazard ratio
$\mathrm{PET}=$ positron emission tomography

records were reviewed for demographic and clinical data, including demographics, preoperative clinical assessment, preoperative radiologic assessment, associated comorbidities, and clinical stage. Records were also reviewed for perioperative and pathologic data, including surgical approach, extent of resection, perioperative mortality (defined as death during the same hospitalization or within 30 days after operation), morbidity, tumor size, histology, pathologic stage, and use of induction or adjuvant therapies. Patients were staged according to the TNM classification of the sixth and seventh editions of American Joint Committee for Cancer staging manuals. ${ }^{10,11}$

\section{Preoperative Evaluation}

Preoperative assessment was directed toward establishing a clinical TNM stage and assessing the patients' ability to tolerate the planned operation. Standard diagnostic and staging workup in this cohort included an upper endoscopy with biopsy and computed tomography (CT) of the chest and upper abdomen to evaluate locoregional extent of the disease and exclude distant metastases. A subset of patients underwent endoscopic ultrasonography and positron emission tomography (PET), which were routinely obtained preoperatively in the latter half of the cohort from 1999 to 2009. Generally, patients were considered for surgical resection if preoperative evaluation revealed no evidence of distant visceral metastases or clear evidence of direct tumor invasion of the airway or major vascular structures. The presence of extensive nodal disease was not considered a contraindication to resection unless it clearly extended beyond the proposed fields of dissection. Performance status of all patients was graded according to Eastern Cooperative Oncology Group guideline. ${ }^{12}$ Finally, all patients underwent detailed evaluation of pulmonary and cardiac function to determine their ability to withstand the planned procedure. Generally, patients with a forced expiratory volume in 1 second less than $1.5 \mathrm{~L} / \mathrm{sec}$ despite aggressive physiotherapy and optimal bronchodilator therapy were considered ineligible for resection. Cardiac disease, if suspected, was carefully assessed using noninvasive stress testing or coronary angiogram if necessary.

\section{Induction and Adjuvant Therapies}

Among all 500 patients, 193 had induction therapy, 179 of whom were completely resected and included with the 465 patients with R- 0 resection. A total of 151 patients received platinum-based induction chemotherapy before surgical resection as part of an ongoing institutional protocol, and 28 patients received induction chemoradiation. A total of 286 patients underwent surgery without preoperative therapy, and 79 of those received adjuvant therapy.

\section{Surgical Resection}

Surgical resection in this cohort of patients consisted of a standard esophagectomy with or without thoracotomy or transthoracic en bloc esophagectomy with 2- or 3-field lymphadenectomy. The basic technique of en bloc esophagectomy has been described. ${ }^{13}$ En bloc esophagectomy was commonly carried out through 3 incisions: a right thoracotomy followed by a laparotomy and collar neck incision. The intrathoracic portion of the en bloc technique included resection of the tumor-bearing esophagus within a wide envelope of periesophageal tissue including both pleural surfaces laterally, a patch of pericardium anteriorly in patients with T2 to T3 tumors, all lymphovascular tissue posterior to the esophagus including the thoracic duct, and a mediastinal lymphadenectomy extending from the tracheal bifurcation to the hiatus. A "third field" lymphadenectomy was incorporated in some patients by extending the nodal dissection to include the superior mediastinal nodes and the nodes along the course of both recurrent laryngeal nerves, and in some patients the deep cervical nodes located lateral and posterior to the jugular veins with the latter performed through the cervical incision. In the abdomen, an upper abdominal lymphadenectomy was performed that included the common hepatic, celiac, left gastric, parahiatal, lesser curvature, and retroperitoneal lymph nodes.

Standard resections in this cohort were done through a transhiatal or transthoracic approach. Transhiatal esophagectomy was performed as described by Orringer and colleagues ${ }^{1}$ through an upper abdominal incision and a left neck incision. However, the parahiatal dissection and upper abdominal nodal dissection were similar to that performed during the abdominal phase of the en bloc operation. The paraesophageal and mediastinal lymph nodes were removed as exposure allowed. During standard transthoracic resections, the esophagus was mobilized with its periesophageal nodes without resection of its pleural envelope, pericardium, thoracic duct, or superior mediastinal lymph nodes. The abdominal dissection was similar to that described previously for en bloc and transhiatal esophagectomy. Generally, a standard esophagectomy was preferentially performed in patients with clinical stage I disease and patients aged 80 years or more. En bloc esophagectomy was performed in most medically fit patients aged less than 80 years with clinical stage II or greater disease. Reconstruction of the gastrointestinal tract was performed using a greater curvature gastric tube in $96 \%$ of patients. Pathologic staging was determined according to the TNM classification of the sixth and seventh editions of the American Joint Committee for Cancer staging manuals. ${ }^{10,11}$

\section{Follow-up}

After hospital discharge, patients were seen at intervals of 3 months for the first 2 years and every 6 months thereafter. Patients from distant geographic regions were followed by contacting their local physician and directly contacting the patient. CT scans of the chest and upper abdomen were obtained every 6 months for 2 years and every year thereafter. Other studies, such as endoscopy and PET scanning, were performed when recurrence was suspected.

\section{Recurrence}

Local recurrence was defined as any tumor recurrence within the field of nodal dissection or at the anastomosis. Distant recurrence was defined as any tumor recurrence occurring in nodal basins outside the field of surgical dissection or in distant organs. The operating surgeon reported recurrence data based on review of findings on CT, PET, endoscopy, or relevant biopsies.

\section{Survival Modeling and Statistical Analysis}

Kaplan-Meier survival analysis of the date of surgery to the date of recurrence, with censoring of all other patients, was used to estimate freedom from recurrence (FFR) after resection. DFS was defined as the date of surgery to the date of recurrence or death from any cause. The log-rank test was used to determine the significance of subgroup comparisons in regard to these survival end points. Median FFR and DFS were computed, and 95\% confidence intervals (CIs) for median FFR/DFS and survival estimates at select time points are presented to assess the precision of the obtained estimates.

Cox proportional hazards regression was used to estimate unadjusted hazard ratios (HRs) for the associations of specific factors of interest and survival outcomes of recurrence and death from any cause. Multivariate Cox proportional hazards models for FFR and DFS were constructed for the analysis of multiple patient characteristics, histopathologic features of the tumor, and treatment regimens. The multivariate regression models 
TABLE 1. Demographic, surgical, and pathologic characteristics of 465 patients who underwent R-0 esophagectomy

\begin{tabular}{|c|c|c|c|c|}
\hline & Entire cohort & En bloc & Standard & $P$ \\
\hline $\mathrm{N}$ & 465 & 328 & 137 & \\
\hline Male & $370(79.6 \%)$ & $267(81.4 \%)$ & $103(75.2 \%)$ & .129 \\
\hline $\begin{array}{l}\text { Age, y (median } \\
\text { value) }\end{array}$ & 64 & 63 & 68 & $<.001$ \\
\hline Performance status 0 & $243(52.3 \%)$ & $173(52.7 \%)$ & $70(51.1 \%)$ & .746 \\
\hline $\begin{array}{l}\text { Performance status } 1 \\
\text { or } 2\end{array}$ & $222(47.7 \%)$ & $155(47.3 \%)$ & $67(48.9 \%)$ & \\
\hline Clinical stage $0 * / I$ & $82(19.5 \%)$ & $33(10.9 \%)$ & $49(41.2 \%)$ & $<.001$ \\
\hline $\begin{array}{l}\text { Clinical stage } \\
\text { II/III/IV }\end{array}$ & $339(80.5 \%)$ & $269(89.1 \%)$ & $70(58.8 \%)$ & \\
\hline $\begin{array}{l}\text { Pathologic stage } \\
\qquad 0^{* / I}\end{array}$ & $117(25.2 \%)$ & $71(21.6 \%)$ & $46(33.6 \%)$ & .007 \\
\hline $\begin{array}{l}\text { Pathologic stage } \\
\text { II/III/IV }\end{array}$ & $348(74.8 \%)$ & $257(78.4 \%)$ & $91(66.4 \%)$ & \\
\hline Adenocarcinoma & $341(73.3 \%)$ & $236(72.0 \%)$ & $105(76.6 \%)$ & .297 \\
\hline $\begin{array}{l}\text { Proximal/mid/lower } \\
\text { third or GEJ }\end{array}$ & $14 / 71 / 380$ & $11 / 55 / 262$ & $3 / 16 / 118$ & .281 \\
\hline Induction therapy & $179(38.5 \%)$ & $159(48.5 \%)$ & $20(14.6 \%)$ & $<.001$ \\
\hline $\begin{array}{c}\text { Perioperative } \\
\text { mortality }\end{array}$ & $16(3.4 \%)$ & $11(3.4 \%)$ & $5(3.6 \%)$ & .284 \\
\hline
\end{tabular}

included age, gender, performance status, extent of resection, induction treatment, cell type, and tumor differentiation. The models were constructed using clinical stage or pathologic stage due to colinearity between the 2 variables, which precluded their inclusion in the models simultaneously. Stage of disease was defined as early stage (0 or I) or advanced stage (II/III/IV). Before the final specification of each multivariate model, potential colinearity between univariate predictors was assessed by the Pearson (or Spearman-rank) correlation coefficient (for continuous predictors) or the kappa statistic (for categoric predictors). The adjusted HRs for recurrence (FFR) and recurrence or death (DFS) are presented along with $95 \%$ CIs for assessing the precision of the obtained HR estimates.

The 2-sample $t$ test or Mann-Whitney $U$ test was used, as appropriate, for the comparison of continuous variables between groups. The chi-square test or Fisher exact test was used, as appropriate, to compare categoric variables between groups. All $P$ values are 2 -sided. All analyses were performed in SPSS Version 18.0 (SPSS Inc, Chicago, Ill).

\section{RESULTS}

\section{Patients}

During the study period from November 1987 to December 2009, 500 consecutive patients with esophageal cancer were surgically treated. Thirty-five patients were excluded from analysis because they had microscopically positive margins $(n=15)$ or gross residual disease $(n=20)$; the latter included 7 patients with unsuspected distant metastases. The overall R0 resection rate was thus $93 \%$. Among the 465 patients who had an R0 resection, 179 were resected after induction therapy and 286 were treated with surgery alone. These completely resected patients comprised the study group and are the subject of all subsequent analyses. Demographic, clinical, and pathologic characteristics of these patients are
TABLE 2. Clinical and pathologic staging of the entire cohort of 465 patients according to pathologic staging per the 6th and 7th editions of the American Joint Committee for Cancer staging manuals

\begin{tabular}{|c|c|c|c|c|c|c|c|}
\hline \multirow{2}{*}{\multicolumn{3}{|c|}{$\begin{array}{c}\text { Clinical stage } \\
\text { 6th ed. }\end{array}$}} & \multirow[b]{3}{*}{ Stage } & \multicolumn{4}{|c|}{ Pathologic stage } \\
\hline & & & & \multicolumn{2}{|c|}{ 6th ed. } & \multicolumn{2}{|c|}{ 7th ed. } \\
\hline Stage & $\mathbf{N}$ & $\%$ & & $\mathbf{N}$ & $\%$ & $\mathbf{N}$ & $\%$ \\
\hline $0^{*}$ & 7 & 1.5 & $0^{*}$ & 20 & 4.3 & 20 & 4.3 \\
\hline I & 75 & 16.1 & I & 97 & 20.9 & & \\
\hline IIA & 118 & 25.4 & IA & & & 70 & 15.1 \\
\hline IIB & 31 & 6.7 & IB & & & 39 & 8.4 \\
\hline III & 155 & 33.3 & IIA & 74 & 15.9 & 23 & 4.9 \\
\hline IVA & 23 & 4.9 & IIB & 67 & 14.4 & 91 & 19.6 \\
\hline IVB & 12 & 2.6 & III & 138 & 29.7 & & \\
\hline \multirow[t]{6}{*}{ Missing } & 44 & 9.5 & IIIA & & & 85 & 18.3 \\
\hline & & & IIIB & & & 65 & 14.0 \\
\hline & & & IIIC & & & 72 & 15.5 \\
\hline & & & IVA & 23 & 4.9 & & \\
\hline & & & IVB & 46 & 9.9 & & \\
\hline & & & Missing & 0 & & & \\
\hline
\end{tabular}

*Clinical stage 0 denotes Tis disease preoperatively, and pathologic stage 0 denotes complete pathologic response after induction therapies.

shown in Table 1. Median age was 64 years (range, 24-89 years), and the majority of patients $(79.6 \%)$ were men.

\section{Surgical Approach and Extent of Resection}

A total of 328 patients $(71 \%)$ had an en bloc resection (2-field in 129, 3-field in 199). The remaining 137 patients $(29 \%)$ had a standard resection (88 transhiatal, 49 transthoracic). Compared with patients treated with standard esophagectomy, those treated by an en bloc resection were significantly younger, had more advanced clinical and pathologic stages, and were more likely to have received preoperative therapy (Table 1 ).

\section{Hospital Morbidity and Mortality}

There were 16 deaths $(3.4 \%)$ that occurred within 30 days from operation or during the same hospitalization. A total of 281 patients $(56 \%)$ had an uncomplicated postoperative course. The most frequent morbidity was pulmonary ( $26 \%$ of patients), followed by cardiac arrhythmia ( $22 \%$ patients). Recurrent nerve injury occurred in $7 \%$ of patients. Anastomotic leaks occurred in $11.5 \%$ of patients. Although slightly more patients had cardiopulmonary morbidity after en bloc resection, only the incidence of atrial arrhythmias was significantly higher after en bloc resection $(P=.01)$. There was also no difference between the 2 approaches in the frequency of chylothoraces, infectious complications, or recurrent nerve injuries. We have previously reported on the morbidity associated with en bloc resections. ${ }^{13,14}$

\section{Induction Therapy}

Of the 179 patients treated preoperatively, 151 had received platinum-based chemotherapy and 28 were treated 
TABLE 3. Crude recurrence rates after en bloc and standard resection in patients treated with surgery alone $(\mathrm{n}=\mathbf{2 8 6})$

\begin{tabular}{|c|c|c|c|c|c|c|c|c|}
\hline \multirow{2}{*}{$\begin{array}{l}\text { Pathologic stage 6th ed. } \\
\text { I }\end{array}$} & \multicolumn{4}{|c|}{ Any recurrence } & \multicolumn{4}{|c|}{ Local recurrence } \\
\hline & Overall & En bloc & Standard & & Overall & En bloc & Standard & \\
\hline & $\mathrm{n}=84$ & $\mathrm{n}=42$ & $\mathrm{n}=42$ & & $\mathrm{n}=84$ & $\mathrm{n}=42$ & $\mathrm{n}=42$ & \\
\hline & $6(7.1 \%)$ & $3(7.1 \%)$ & $3(7.1 \%)$ & $P=1.00$ & $1(1.2 \%)$ & $1(2.4 \%)$ & 0 & $P=1.00$ \\
\hline \multirow[t]{3}{*}{ II/III/IV } & Overall & En bloc & Standard & & Overall & En bloc & Standard & \\
\hline & $\mathrm{n}=202$ & $\mathrm{n}=127$ & $\mathrm{n}=75$ & & $\mathrm{n}=202$ & $\mathrm{n}=127$ & $\mathrm{n}=75$ & \\
\hline & $102(50.5 \%)$ & $63(49.6 \%)$ & $39(52.0 \%)$ & $P=.742$ & $27(13.4 \%)$ & $17(13.4 \%)$ & $10(13.3 \%)$ & $P=.992$ \\
\hline
\end{tabular}

with preoperative chemoradiation. The latter half of the cohort included 51.5\% (163/319) who received preoperative therapy from 1999 to 2009 , compared with $11.0 \%$ $(16 / 146)$ in the earlier half of the cohort treated from 1987 to $1998(P<.001)$.

\section{Pathologic Findings}

Clinical and pathologic staging of the entire cohort is detailed in Table 2, which was determined according to the sixth and seventh editions of American Joint Committee for Cancer staging manuals. ${ }^{10,11}$ At the time of surgical resection, slightly less than half of the patients $(44.5 \%)$ had pathologic stage III and IV disease. The most common tumor cell type was adenocarcinoma (73.3\%), and most tumors were located in the lower third of the esophagus (Table 1). The median number of lymph nodes removed among all patients was 26 . The number of harvested lymph nodes was significantly higher for the en bloc group compared with the standard group (median 31 vs $17, P<.001)$. A total of 265 patients $(57 \%)$ had pathologically involved lymph nodes. The median number of positive lymph nodes was 3 regardless of the extent of dissection. After induction therapy, 20 patients $(10 \%)$ had a complete pathologic response.

\section{Predictors of Freedom From Recurrence}

Median follow-up for all surviving patients was 49 months. Of the 465 patients who had an R-0 resection, tumor recurrence developed in 197 (42.4\%). Among 286 patients treated by surgery without induction therapy, local failure as previously defined was $10.7 \%$ after en bloc resection and $8.5 \%$ after standard resection, for an overall local recurrence rate of $9.8 \%$. Table 3 illustrates stage matched comparisons of overall and local recurrence rates for early and advanced disease, which demonstrate no difference between the 2 resection techniques. These results were unchanged when the crude recurrence rates were calculated for the entire cohort of 465 patients who had an R-0 resection (data not shown).

For patients with early pathologic stage disease, there was no difference between the en bloc and standard techniques in FFR for the entire cohort (Figure 1) and for those patients treated by surgery alone (Figure 2). In patients with locally advanced stage disease treated by surgery alone, en bloc resection (compared with standard resection) was asso- ciated with a significant improvement in FFR (Figure 2); the median FFR after en bloc and standard resections was 32.4 months (95\% CI, 10.3-54.5) and 20.7 months (95\% CI, 11.7-29.8), respectively (HR, 0.64; CI, 0.43-0.96; $P=.028)$. However, when all patients with advanced pathologic stage disease were analyzed, including patients treated with induction therapy (Figure 1), there was a nonsignificant trend in favor of en bloc resection with a median time for FFR of 25.0 months (95\% CI, 16.8-33.2) versus 20.7 months (95\% CI, 13.2-28.3) after standard resection (HR, 0.82; CI, 0.58-1.14; $P=.237$ ). Results were the same when analysis of FFR was repeated using the AJCC Cancer Staging Manual, 7 th ed. ${ }^{11}$

To further elucidate independent predictors of FFR, multivariate regression analysis was also performed with variables thought to be associated with FFR. The model demonstrated that performance status, tumor differentiation, extent of resection, and use of induction therapy were significant independent predictors of FFR, in addition to pathologic stage (Table 4). Results were similar when the model was adjusted for clinical stage, with the exception of induction therapy, which was no longer a significant predictor of FFR in this model (Table 5).

\section{Predictors of Disease-Free Survival}

For the 465 patients in whom an R-0 resection was performed, 5-year DFS was 39.8\% (95\% CI, 34.9-44.7) and 10-year DFS was 31.8\% (95\% CI, 26.5-37.1). For patients with clinical stage $0 /$ I disease, ${ }^{10}$ there was no significant difference in DFS between the en bloc group and the standard resection group (Figure 3). However, for patients with clinical stage II or greater disease, DFS was significantly prolonged after en bloc esophagectomy. Median DFS was 23.6 months (95\% CI, 17.8-29.4) after en bloc resection and 12.2 months $(95 \% \mathrm{CI}, 5.2-19.2)$ after standard resection (HR, 0.70; CI, 0.50-0.97; $P=.033$ ).

For patients with pathologic stage $0 /$ I disease, ${ }^{10}$ there was again no significant difference in DFS between the en bloc group and the standard resection group (5 year-DFS 75.7\% [95\% CI, 62.2-90.4] vs 76.3\% [95\% CI, 65.386.1]; Figure 4). However, for patients with pathologic stage II/III/IV disease, DFS was significantly improved after en bloc resection compared with standard resection (HR, 0.66; CI, 0.50-0.88; $P=.004)$. Median DFS was 19.0 months (95\% CI, 14.0-24.0) after en bloc and 12.2 months 


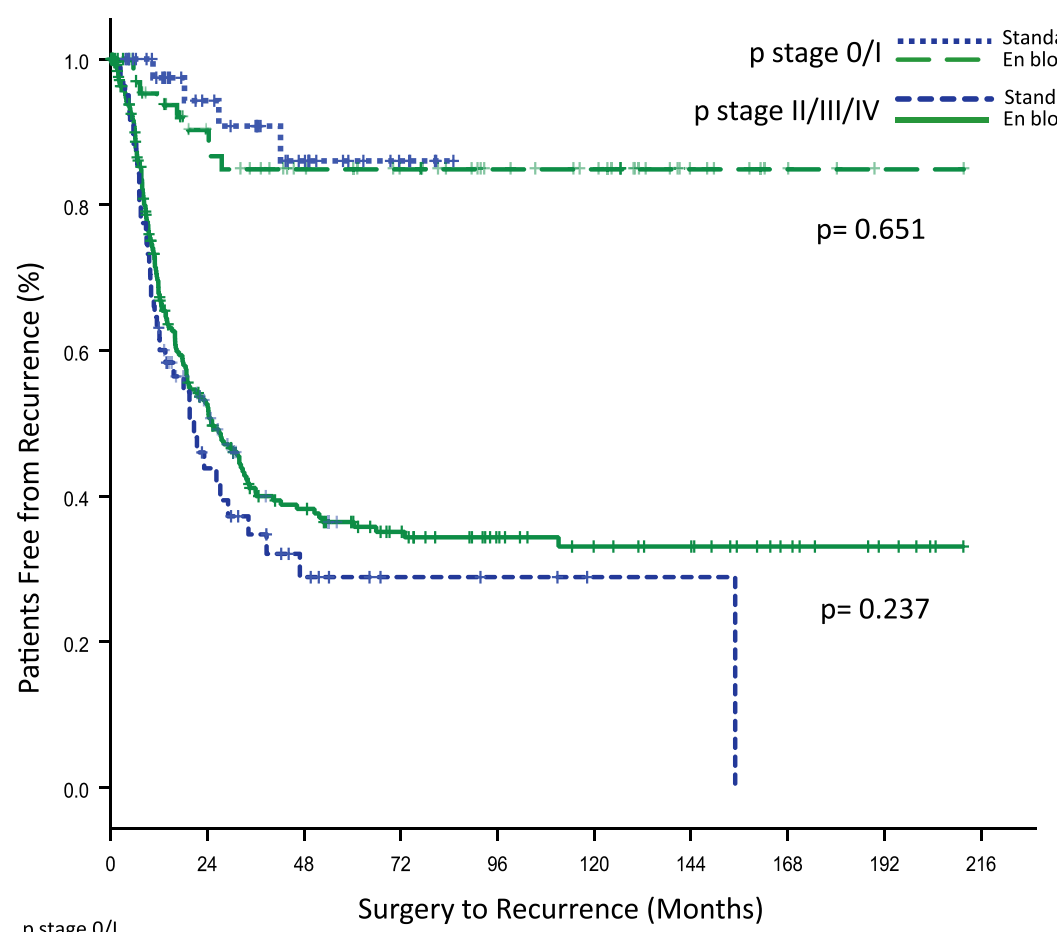

\begin{tabular}{cccccccccc} 
At risk & p stage 0/I & \multicolumn{8}{c}{ Surgery to Recurrence (Months) } \\
En bloc & 71 & 50 & 40 & 35 & 26 & 22 & 9 & 4 & 0 \\
Standard & 46 & 28 & 15 & 5 & 0 & & & & \\
& p stage II/III/IV & & & & & & & & \\
En bloc & 257 & 106 & 65 & 48 & 34 & 24 & 21 & 11 & 5 \\
Standard & 91 & 20 & 9 & 4 & 3 & 1 & 0 & &
\end{tabular}

FIGURE 1. FFR for the whole cohort, stratified as early (stage 0/I) or advanced (stage II/III/IV) pathologic stage.

(95\% CI, 7.7-16.7) after standard resection. When patients were restaged by criteria of the AJCC Cancer Staging Man$u a l, 7$ th ed, ${ }^{11}$ similar results were observed (data not shown).

Multivariate analysis of DFS revealed that the extent of resection, performance status, and use of induction therapy were significant independent predictors of DFS (Table 6). However, because a significant interaction between pathologic stage and extent of resection was observed (interaction coefficient $P=.054$ ), the multivariate model was stratified by early or advanced pathologic stage. Because of the relatively small numbers and infrequency of events in the early-stage subgroup, there was instability in the model estimates and the data are not shown (ie, the multivariate model as originally specified for DFS could not be supported in the early-stage subgroup). Analysis of the advanced stage subgroup is shown in Table 7. The extent of resection and performance status emerged as significant independent factors, as they had when the entire cohort was analyzed, whereas the association between induction therapy and worse DFS was not significant.

When the model was adjusted for clinical stage instead of pathologic stage, performance status, extent of resection, and tumor differentiation emerged as significant independent predictors of DFS, whereas induction therapy was no longer a significant predictor (Table 8). When the interaction between extent of resection and clinical stage was tested in this model, there was no evidence of interaction between these 2 variables (interaction coefficient $P=.911$ ).

\section{DISCUSSION}

In contrast with resectional surgery for malignant disease in organs such as the lung, colon, and breast, esophagectomy for esophageal carcinoma remains a nonstandardized operation in almost every respect other than its basic concept. Surgeons around the world performing the operation are properly influenced by prior training, personal experience, and often strongly held beliefs about the "biology" of this neoplasm. In the absence of standardization of the technique of resection, particularly as it relates to the extent of nodal dissection, it is not possible to rationally compare outcomes from various centers, let alone evaluate the impact of various induction therapy protocols. In the current work, our aim was to examine the factors influencing disease recurrence and DFS in a cohort of patients treated by surgical techniques that have been well standardized and uniformly applied in a single institution over the past 2 decades. Our results show that, for both FFR and DFS, outcome is often dictated by multiple factors that include patient-related variables, tumor-related factors, and treatment-related factors, including the technique of surgical resection. The results pertaining to disease recurrence are of particular interest because we and other advocates 


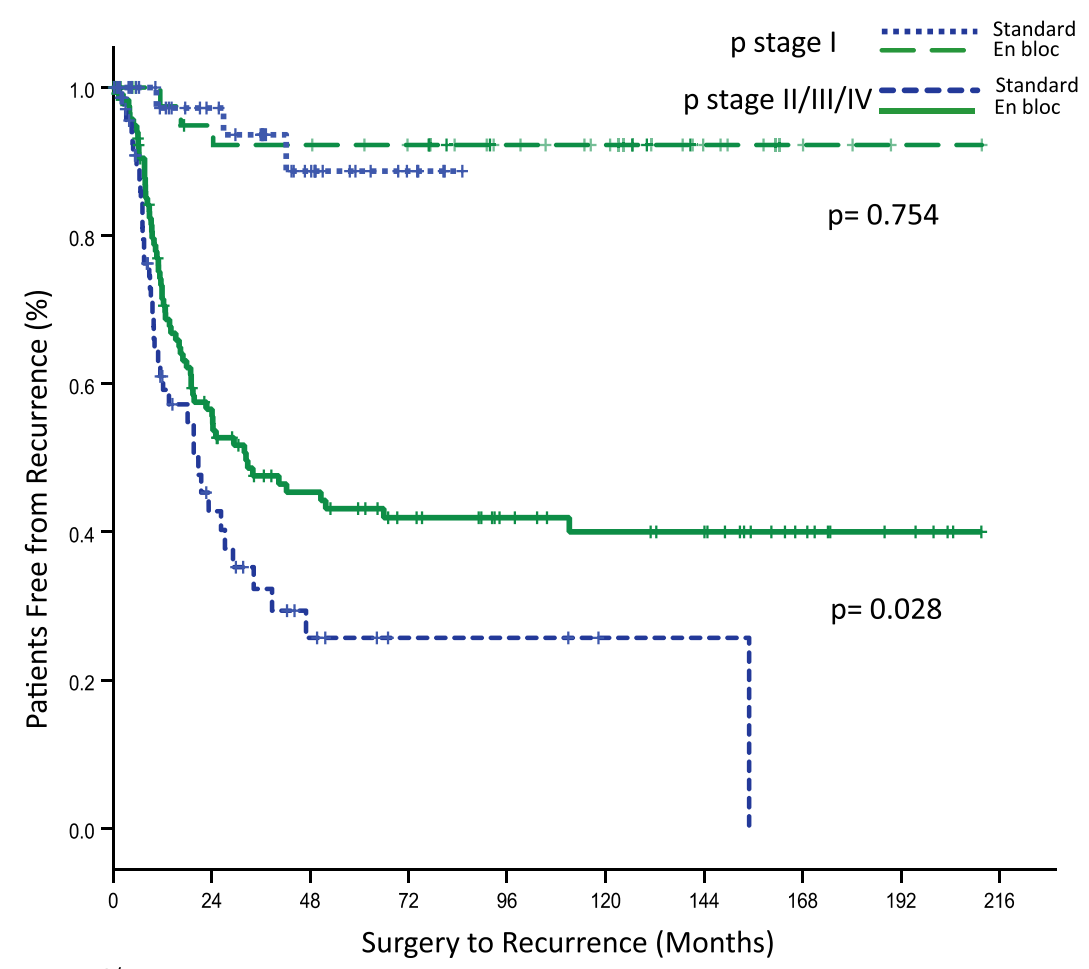

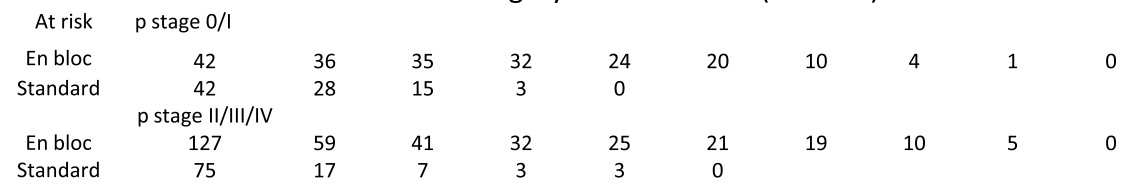

FIGURE 2. FFR for patients treated with surgery alone, stratified as early (stage I) or advanced (stage II/III/IV) pathologic stage.

of the en bloc technique have argued that one mechanism by which en bloc resection improves survival may be through delivery of superior local disease control. ${ }^{13,15,16}$ Previously reported locoregional failure rates after en bloc esophagectomy compared favorably with historical local failure rates after transthoracic or transhiatal esophagectomy, which have been variably reported to be in the $30 \%$ to $40 \%$ range..$^{6-9}$ Although the use of induction chemoradiation may have provided better local control, local recurrence rates after transhiatal resection remained high at approximately $20 \%{ }^{7}$ Notably, the current analyses show no significant difference in locoregional

TABLE 4. Multivariate Cox regression, freedom from recurrence adjusted for pathologic stage

\begin{tabular}{lcr}
\hline & HR & \multicolumn{1}{c}{$\boldsymbol{P}$} \\
\hline Age & $0.99(0.97-1.00)$ & .073 \\
Male gender & $1.28(0.87-1.90)$ & .211 \\
Performance status $>0$ & $1.84(1.35-2.49)$ & $<.001$ \\
Poor differentiation & $1.50(1.12-2.01)$ & .006 \\
Squamous cell type & $1.03(0.74-1.44)$ & .845 \\
Induction & $1.65(1.21-2.25)$ & .002 \\
En bloc resection & $0.61(0.43-0.88)$ & .007 \\
Advanced pathologic stage & $5.46(3.05-9.78)$ & $<.001$ \\
\hline
\end{tabular}

$H R$, Hazard ratio. crude recurrence rates between patients treated with or without en bloc resection. The local recurrence rates reported in this article are essentially similar to those reported by Hulscher and colleagues ${ }^{17}$ in a randomized trial comparing transhiatal resection with transthoracic en bloc esophagectomy. These results, though interesting, should be interpreted within the context of 2 important considerations. First, approximately $40 \%$ of patients treated by standard resections had clinical stage $0 / \mathrm{I}$ disease compared with only $10 \%$ of patients in the en bloc group, a highly significant difference. Conversely, approximately $80 \%$ of the en bloc group had pathologically staged locally advanced

TABLE 5. Multivariate Cox regression, freedom from recurrence adjusted for clinical stage

\begin{tabular}{lcr}
\hline & HR & \multicolumn{1}{c}{$\boldsymbol{P}$} \\
\hline Age & $0.99(0.97-1.00)$ & .157 \\
Male gender & $1.29(0.85-1.95)$ & .233 \\
Performance status $>0$ & $2.02(1.47-2.77)$ & $<.001$ \\
Poor differentiation & $1.74(1.28-2.38)$ & $<.001$ \\
Squamous cell type & $0.84(0.59-1.20)$ & .332 \\
Induction & $1.35(0.98-1.87)$ & .067 \\
En bloc resection & $0.60(0.40-0.90)$ & .014 \\
Advanced clinical stage & $6.46(2.90-14.38)$ & $<.001$ \\
\hline
\end{tabular}

$H R$, Hazard ratio. 


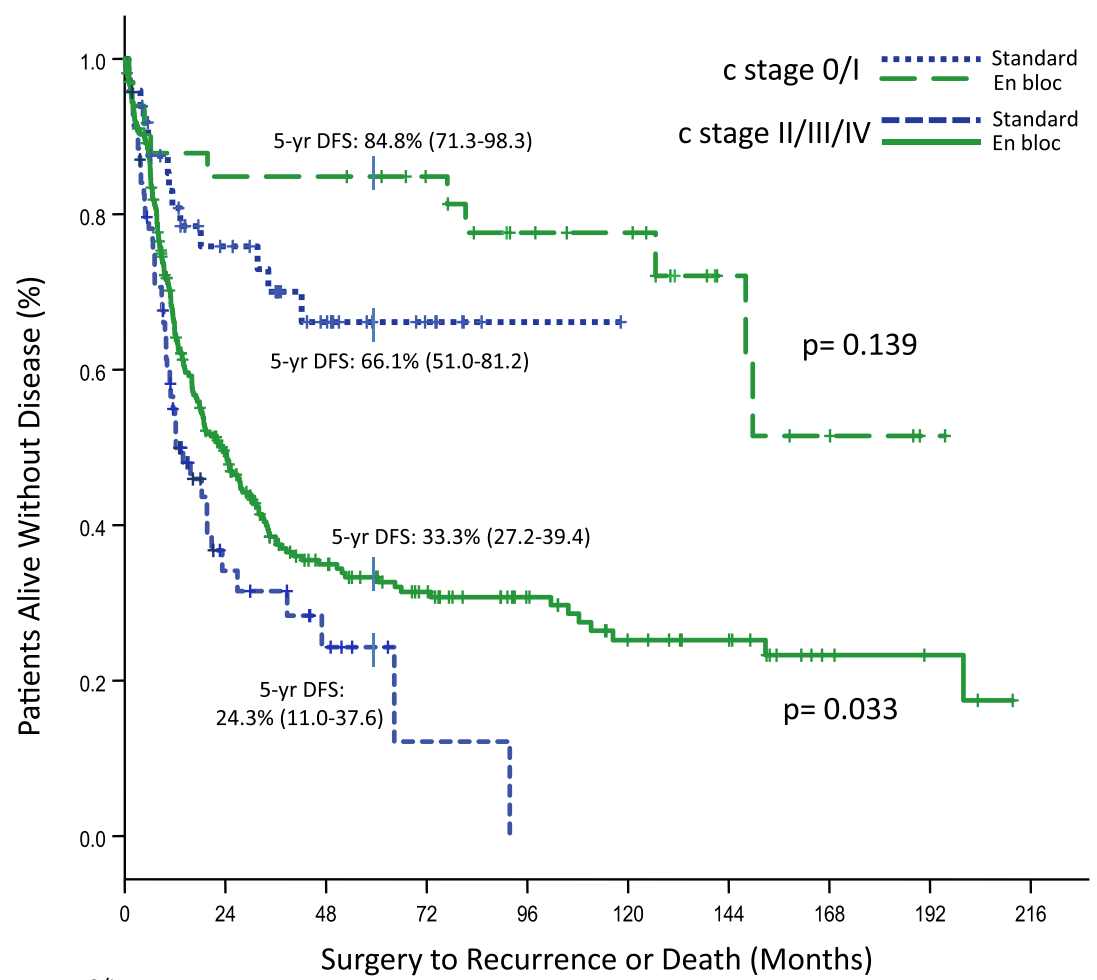

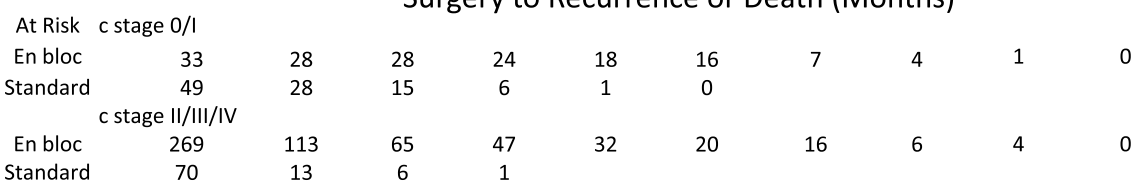

FIGURE 3. DFS for the entire cohort, stratified as early (stage 0/I) or advanced (stage II/III/IV) clinical stage.

disease despite the use of preoperative therapy in approximately half the patients. Second, during the conduct of the transhiatal resection we have essentially performed an upper abdominal node dissection that is identical to that carried out during the abdominal phase of the en bloc operation. The median number of lymph nodes resected in the standard resection group was 17 , a number that compares favorably with published recommendations for adequate nodal staging, and therefore may have contributed to some extent in decreasing local failure in the upper abdomen. ${ }^{18}$

Despite the similarity in the crude rates of local recurrence, it seems there is a significant difference in the FFR between the 2 procedures. Patients with advanced pathologic stage who were treated by surgery alone had a significant $36 \%$ reduction in the hazard for recurrence after en bloc resection and an increase in the FFR by approximately 1 year. Although there was a similar trend favoring en bloc resection when all patients with advanced pathologic stages were analyzed (including patients treated by neoadjuvant therapy), statistical significance was lost probably because the magnitude of the beneficial effect was reduced by the inclusion of a group of patients with biologically more virulent disease all treated by en bloc resection. This is consistent with the pathologic stage-adjusted multivariable model of the entire cohort, which showed that en bloc resection reduced the hazard for recurrence, whereas induction therapy was significantly associated with recurrent disease. It is obviously of equal interest that the FFR did not differ significantly between the 2 resection techniques in patients with early-stage disease (cT1N0/pT1N0) or those downstaged to ypT0-1/N0 after preoperative therapy.

This report also analyzed the factors that may be associated with prolonged DFS. Only performance status, extent of resection, and stage consistently emerged from the Cox regression models as independent predictors of DFS. To further define the role of surgical resection, we examined DFS after the 2 types of resection in various clinical and pathologic stage groupings. Our results suggest that patients with early-stage disease (cT1N0/pT1N0) can be appropriately and sufficiently treated by an en bloc or a standard resection. Conversely, the data also suggest that an en bloc resection results in a significant improvement in DFS compared with standard resection in patients with advanced clinical or pathologically staged disease. In the absence of a demonstrable effect on local failure rates, the therapeutic benefit of en bloc resection may be derived from a wider lymphadenectomy with excision of both clinically apparent 

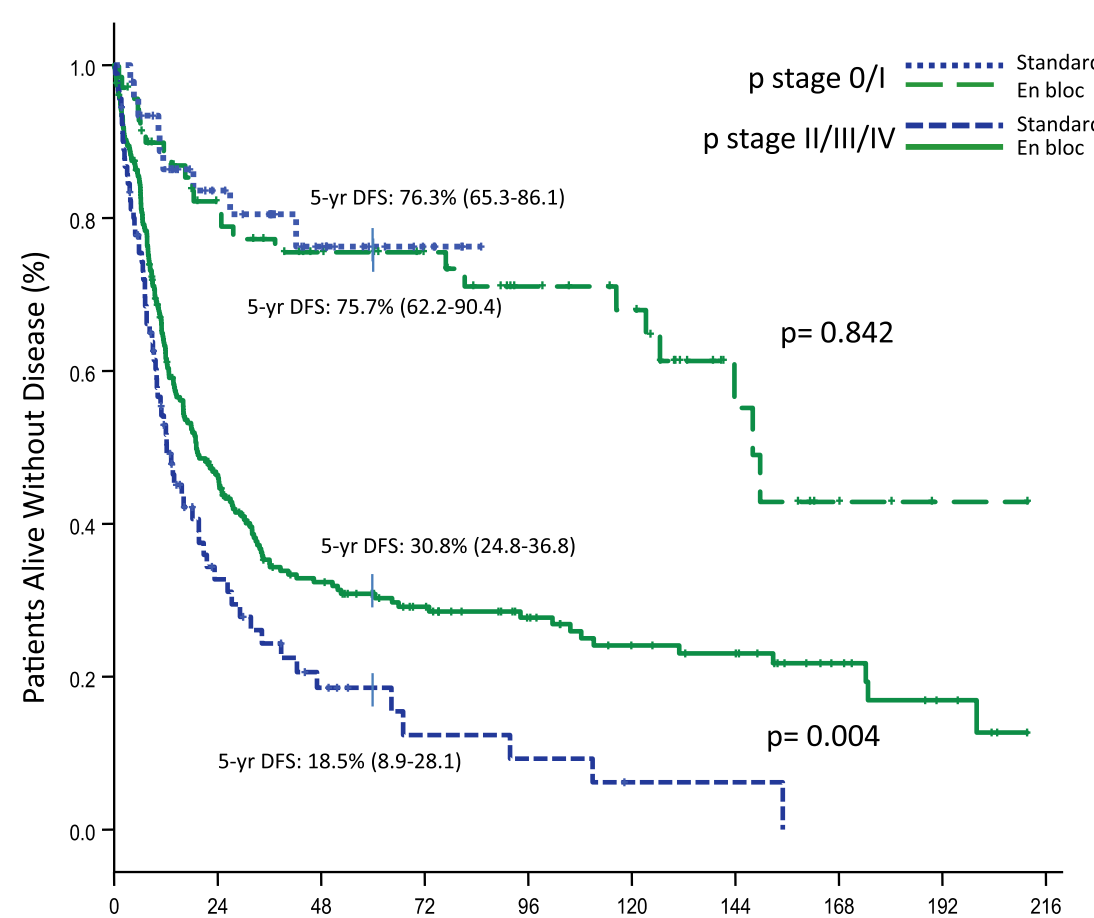

Surgery to Recurrence or Death (Months)

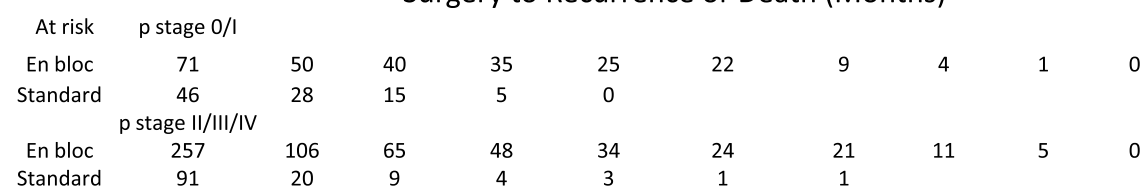

FIGURE 4. DFS for the entire cohort, stratified as early (stage 0/I) or advanced (stage II/III/IV) pathologic stage (AJCC Cancer Staging Atlas, 6th ed ${ }^{10}$ ).

and occult nodal metastases. We previously reported that the total number of resected nodes is a significant determinant of survival in node-positive patients treated by surgery alone. ${ }^{19}$ In comparison with patients with 16 or fewer lymph nodes resected, the HR for death was significantly reduced to 0.51 when 17 to 25 nodes were resected and 0.39 when 26 to 40 nodes were removed. Recent reviews of the Surveillance, Epidemiology, and End-Results database and of an international database reported increased survival with higher total negative lymph node counts and higher total lymph node counts. $^{20,21}$ Thus, extended lymphadenectomy, beyond its influence on stage migration, may have a therapeutic

TABLE 6. Multivariate Cox regression, disease-free survival adjusted for pathologic stage

\begin{tabular}{lcr}
\hline & HR & \multicolumn{1}{c}{$\boldsymbol{P}$} \\
\hline Age & $1.00(0.99-1.01)$ & .575 \\
Male gender & $1.16(0.85-1.58)$ & .360 \\
Performance status $>0$ & $1.73(1.34-2.23)$ & $<.001$ \\
Poor differentiation & $1.25(0.98-1.60)$ & .069 \\
Squamous cell type & $1.25(0.94-1.67)$ & .120 \\
Induction & $1.34(1.02-1.76)$ & .033 \\
En bloc resection & $0.63(0.47-0.84)$ & .002 \\
Advanced pathologic stage & $3.16(2.15-4.65)$ & $<.001$ \\
\hline
\end{tabular}

$H R$, Hazard ratio. benefit by removing lymph nodes containing occult metastatic nodal disease. Extended lymphadenectomy may be important even after induction therapy, because in the majority of patients neither preoperative chemotherapy nor preoperative chemoradiation reliably eliminates nodal disease. $^{22}$ The controversy around the extent of lymph node dissection for cancer has occupied esophageal surgeons for decades, and the results of a recent randomized trial has done little to quell the controversy. ${ }^{17}$ In that trial, 220 patients were randomly assigned to en bloc or transhiatal esophagectomy. Although the results tantalizingly showed a 34\% relative improvement in both overall and DFS after en bloc

TABLE 7. Multivariate Cox regression, disease-free survival in patients with advanced pathologic stages

\begin{tabular}{lcr}
\hline & HR & \multicolumn{1}{c}{$\boldsymbol{P}$} \\
\hline Age & $1.00(0.99-1.01)$ & .653 \\
Male gender & $1.17(0.83-1.63)$ & .365 \\
Performance status $>0$ & $1.80(1.36-2.37)$ & $<.001$ \\
Poor differentiation & $1.26(0.98-1.63)$ & .077 \\
Squamous cell type & $1.29(0.96-1.75)$ & .094 \\
Induction & $1.28(0.96-1.70)$ & .091 \\
En bloc resection & $0.56(0.41-0.76)$ & $<.001$ \\
\hline
\end{tabular}

$H R$, Hazard ratio. 
TABLE 8. Multivariate Cox regression, disease-free survival adjusted for clinical stage

\begin{tabular}{lcr}
\hline & HR & \multicolumn{1}{c}{$\boldsymbol{P}$} \\
\hline Age & $1.00(0.99-1.02)$ & .761 \\
Male gender & $1.14(0.81-1.60)$ & .465 \\
Performance status $>0$ & $1.95(1.49-2.57)$ & $<.001$ \\
Poor differentiation & $1.42(1.09-1.86)$ & .009 \\
Squamous cell type & $1.06(0.78-1.46)$ & .693 \\
Induction & $1.22(0.91-1.62)$ & .178 \\
En bloc resection & $0.62(0.45-0.87)$ & .006 \\
Advanced clinical stage & $2.75(1.71-4.41)$ & $<.001$ \\
\hline
\end{tabular}

$H R$, Hazard ratio.

esophagectomy ( $39 \%$ en bloc vs $29 \%$ transhiatal), statistical significance was not achieved. The authors subsequently published a subgroup analysis and showed that the long-term benefit of an en bloc resection could be attributed to the subgroup of patients with lower third carcinoma of the esophagus, in whom the estimated 5-year survival benefit of an en bloc resection was $14 \% .^{23}$ The authors also showed that for patients with limited nodal disease (1-8 positive lymph nodes) DFS was significantly improved after en bloc resection. ${ }^{24}$ In the absence of another more definitive randomized trial, this controversy is likely to continue.

\section{Limitations}

Despite our best efforts to collect the data prospectively, this study remains a retrospective review and thus subject to all the inherent limitations of such reviews. For example, unintended biases such as selection or referral bias cannot be reasonably excluded. This study also extended over a 22-year period, during which there has been evolution in staging techniques and multimodality treatment of esophageal cancer. More recently, there has been an obvious shift toward increasing use of PET scanning in preoperative staging and of preoperative chemotherapy or chemoradiation.

\section{CONCLUSIONS}

Our results suggest that, for patients with good performance status and clinically staged as stage II or greater, en bloc resection is associated with a higher probability of FFR and a significant improvement in DFS. Patients with clinical stage I may be adequately treated by a standard resection technique.

\section{References}

1. Orringer MB, Marshall B, Iannettoni MD. Transhiatal esophagectomy: clinical experience and refinements. Ann Surg. 1999;230:392-403.

2. Ellis FH Jr, Heatley GJ, Krasna MJ, Williamson WA, Balogh K. Esophagogastrectomy for carcinoma of the esophagus and cardia: a comparison of findings and results after standard resection in three consecutive eight-year intervals with improved staging criteria. J Thorac Cardiovasc Surg. 1997;113:836-48.

3. Lieberman MD, Shriver CD, Bleckner S, Burt M. Carcinoma of the esophagus. prognostic significance of histologic type. J Thorac Cardiovasc Surg. 1995;109:130-9.

4. Putnam JB Jr, Suell DM, McMurtrey MJ, Ryan MB, Walsh GL, Natarajan G, et al. Comparison of three techniques of esophagectomy within a residency training program. Ann Thorac Surg. 1994;57:319-25.
5. Herskovic A, Martz K, al-Sarraf M, Leichman L, Brindle J, Vaitkevicius V, et al. Combined chemotherapy and radiotherapy compared with radiotherapy alone in patients with cancer of the esophagus. N Engl J Med. 1992;326:1593-8.

6. Altorki NK. The rationale for radical resection. Surg Oncol Clin N Am. 1999;8: 295-305.

7. Urba SG, Orringer MB, Turrisi A, Iannettoni M, Forastiere A, Strawderman M. Randomized trial of preoperative chemoradiation versus surgery alone in patients with locoregional esophageal carcinoma. J Clin Oncol. 2001;19:305-13.

8. Kelsen DP, Ginsberg R, Pajak TF, Sheahan DG, Gunderson L, Mortimer J, et al. Chemotherapy followed by surgery compared with surgery alone for localized esophageal cancer. N Engl J Med. 1998;339:1979-84.

9. Law S, Fok M, Chow S, Chu KM, Wong J. Preoperative chemotherapy versus surgical therapy alone for squamous cell carcinoma of the esophagus: a prospective randomized trial. J Thorac Cardiovasc Surg. 1997;114:210-7.

10. Greene FL, Compton CC, Fritz AG, Shah JP, Winchester DP, Compton CC, et al. AJCC Cancer Staging Atlas. 6th ed. New York, NY: Springer; 2006.

11. Edge SB, Byrd DR, Compton CC, Fritz AG, Greene FL, Trotti A, eds. AJCC Cancer Staging Manual. 7th ed. New York, NY: Springer; 2010.

12. Oken MM, Creech RH, Tormey DC, Horton J, Davis TE, McFadden ET, et al. Toxicity and response criteria of the eastern cooperative oncology group. Am J Clin Oncol. 1982;5:649-55.

13. Altorki N, Skinner D. Should en bloc esophagectomy be the standard of care for esophageal carcinoma? Ann Surg. 2001;234:581-7.

14. Altorki N, Kent M, Ferrara C, Port J. Three-field lymph node dissection for squamous cell and adenocarcinoma of the esophagus. Ann Surg. 2002;236:177-83.

15. Lerut T, Nafteux P, Moons J, Coosemans W, Decker G, De Leyn P, et al. Threefield lymphadenectomy for carcinoma of the esophagus and gastroesophageal junction in 174 R0 resections: Impact on staging, disease-free survival, and outcome: a plea for adaptation of TNM classification in upper-half esophageal carcinoma. Ann Surg. 2004;240:962-74.

16. Rizzetto C, DeMeester SR, Hagen JA, Peyre CG, Lipham JC, DeMeester TR. En bloc esophagectomy reduces local recurrence and improves survival compared with transhiatal resection after neoadjuvant therapy for esophageal adenocarcinoma. J Thorac Cardiovasc Surg. 2008;135:1228-36.

17. Hulscher JB, van Sandick JW, de Boer AG, Wijnhoven BP, Tijssen JG, Fockens P, et al. Extended transthoracic resection compared with limited transhiatal resection for adenocarcinoma of the esophagus. N Engl J Med. 2002;347:1662-9.

18. Rizk N, Venkatraman E, Park B, Flores R, Bains MS, Rusch V, et al. The prognostic importance of the number of involved lymph nodes in esophageal cancer: Implications for revisions of the American Joint Committee on Cancer Staging system. J Thorac Cardiovasc Surg. 2006;132:1374-81.

19. Altorki NK, Zhou XK, Stiles B, Port JL, Paul S, Lee PC, et al. Total number of resected lymph nodes predicts survival in esophageal cancer. Ann Surg. 2008; 248:221-6.

20. Schwarz RE, Smith DD. Clinical impact of lymphadenectomy extent in resectable esophageal cancer. J Gastrointest Surg. 2007;11:1384, 94.

21. Peyre CG, Hagen JA, DeMeester SR, Altorki NK, Ancona E, Griffin SM, et al. The number of lymph nodes removed predicts survival in esophageal cancer: An international study on the impact of extent of surgical resection. Ann Surg. 2008;248:549-56

22. Prenzel KL, Konig A, Schneider PM, Schnickmann C, Baldus SE, Schroder W, et al. Reduced incidence of nodal micrometastasis after major response to neoadjuvant chemoradiation in locally advanced esophageal cancer. Ann Surg Oncol. 2007; 14:954-9.

23. Hulscher JB, van Lanschot JJ. Individualised surgical treatment of patients with an adenocarcinoma of the distal oesophagus or gastro-oesophageal junction. Dig Surg. 2005;22:130-4.

24. Omloo JM, Lagarde SM, Hulscher JB, Reitsma JB, Fockens P, van Dekken H, et al. Extended transthoracic resection compared with limited transhiatal resection for adenocarcinoma of the mid/distal esophagus: five-year survival of a randomized clinical trial. Ann Surg. 2007;246:992-1001.

\section{Discussion}

Dr David Rice (Houston, Tex). I have nothing to disclose.

Congratulations on an excellent presentation, Paul, and thank you very much for giving me the slides and the manuscript well in advance of the meeting.

Dr Lee and his colleagues from Cornell present an impressive series of 500 patients who underwent an esophagectomy for 
esophageal cancer over a 22-year period. The majority of patients underwent surgery alone, but a third also received induction chemotherapy. Two thirds had an en bloc esophagectomy with either a 2-field or 3-field lymphadenectomy. Of the remaining one third, the majority of these had a transhiatal resection. Consistent with most other large Western series, adenocarcinoma predominated and over $80 \%$ of cases were clinical stage II or higher. I applaud the authors on such a low mortality rate.

There is much controversy regarding the oncologic adequacy of transhiatal esophagectomy. Proponents argue that no randomized trial has shown significant inferiority in terms of survival, and certainly pulmonary morbidity is lessened. What is beyond doubt, however, is that transhiatal esophagectomy compromises on the extent of mediastinal node dissection that is achievable. Whether or not this influences local recurrence and survival is the subject of this manuscript. In multivariate analysis, Lee and colleagues found that en bloc esophagectomy was an independent predictor of longer disease-free survival. The survival benefit of en bloc esophagectomy compared with the standard approach was especially marked for patients with stage III and stage IV tumors. Nevertheless, there was only a marginal difference in the proportion of isolated local recurrences that favored the en bloc approach by only 3 percentage points.

My first question to Dr Lee and his colleagues is whether factors other than a less extensive nodal dissection could have resulted in the worse disease-free survival seen with a standard esophagectomy. Is it possible that patients who did not undergo en bloc esophagectomy had factors that would have predicted poor outcome anyway and that we may be dealing with selection bias? Furthermore, was there any difference in cancer-specific death between the standard and the en bloc groups?

Dr Lee. Thank you, Dr Rice, for those very thoughtful questions and your comments.

To answer your question, we did look at the performance status between the en bloc group and the non-en bloc group and there was no difference in terms of performance status. Each group was pretty much evenly split with PS0 and PS1. It is true that the en bloc group had younger patients. The median age was 63 as opposed to 68 . And when we did look at comorbidities, there seemed to be a high incidence of severe pulmonary artery disease in the transhiatal group. Nonetheless, we did look at the cancerspecific survival and we excluded those patients who died from other causes in the analysis. We found that for stage I there was no difference; however, for stage II, III, and IV, there was a significant difference in improved cancer-specific survival in the en bloc group in contrast to the non-en bloc group.

Dr Rice. My second question pertains to the extent of lymphadenectomy required. As you know, the trial by Hulscher and colleagues, which is a randomized trial comparing en bloc esophagectomy to transhiatal esophagectomy, although there was no significant survival difference shown overall, when you broke down patients into whether or not they were N0 versus patients who had 1 to 8 positive nodes and patients who had greater than 8 positive nodes, there actually was a statistically significant improvement in survival favoring the en bloc group for the group of patients who had 1 to 8 positive nodes. In your analysis, were you able to find any subpopulations of patients who seemed to derive the most benefit from the enbloc approach and, if so, are there any preoperative indicators that predict which patients might benefit from a more extensive node dissection?

Dr Lee. Dr Altorki had published in 2001 in the Annals of Surgery that looked at the number of positive lymph nodes and the patients who had more than 7 positive lymph nodes, again, under $\mathrm{pN} 3$ in the new staging system, those patients who had undergone en bloc esophagectomy did a lot worse. So it seems if the patient has anywhere from 1 to 6 positive lymph nodes, that patient probably gleans the most benefit from en bloc resection. But, as you know, it is very difficult, even with endoscopic ultrasound and PET scan, to tell precisely how many lymph nodes are involved, and we struggle with the clinical staging under the new staging system with that question.

Dr Rice. Lastly, I was struck by how few patients underwent preoperative chemoradiation. Any potential benefit of induction chemotherapy is likely due to the eradication of systemic micrometastases. Perhaps in the setting of en bloc esophagectomy, the value of preoperative radiation would be negated. However, induction chemoradiation is, rightly or wrongly, a widely accepted treatment regimen in North America. How do you think your results should be interpreted by surgeons who are advocates of preoperative chemoradiation?

Thank you very much.

Dr Lee. In our study, $80 \%$ of patients who had a recurrence actually recurred distantly. So it's hard to imagine, given an en bloc resection, to give another local therapy 4 or 5 weeks before an en bloc esophagectomy. You are mainly just targeting less than $10 \%$ of the patients. For the remaining $90 \%$ of the patients, you are compromising probably the only treatment, which is chemotherapy, to address the systemic disease issues.

Dr Rice. Thank you very much. I greatly enjoyed the presentation. Congratulations.

Dr Scott Swanson (Boston, Mass). I was interested in your local recurrence rate. If I added it up correctly, it was 9 plus 4 , or about $13 \%$, and in our published series in 2001 from the Brigham, we had about a $3 \%$ local recurrence. Can you tell us a little more about where it occurred and why you think it did? Was it less common in the en bloc patients?

Dr Lee. We define local recurrence as any recurrence within a field dissection. So, for the patient who has a 3-field lymphadenectomy and recurs in the neck, we count that as a local recurrence. I agree that the isolated local recurrence is $9 \%$, and when you put the two together, the recurrence rate is $13 \%$, but bear in mind that I think that the patients we are doing en bloc on are patients who have locally advanced disease and, comparing it to conventional series, the recurrence rate is reported anywhere from $30 \%$ to as high as $60 \%$ in traditional surgical series. I applaud your results in achieving a very low local recurrence rate but I think the $13 \%$ is pretty low when we compare it with the conventional esophagectomies.

Dr Antoon Lerut (Leuven, Belgium). Thank you. These are excellent results indeed.

I would just like to indicate that there is our own experience and also from The Netherlands that, in fact, there is a direct correlation between complications and recurrences. I assume that the patients who have a lesser performance status are those who are more likely to have complications. So I assume that it is not so much the performance status but rather the complications-linked, of course, to a lesser performance status-that are in fact the reason for the 
higher recurrence rate. Have you checked that particular issue? And related to that question is what do you do with the patient with a lesser performance status who is presenting with a stage III carcinoma? Are you going to operate?

Dr Lee. When we look at our overall mortality, there is no difference between the en bloc and the non-en bloc group. It is between $3 \%$ and $4 \%$. We have not looked at the postoperative complications in this study but we do recognize in other series that the en bloc group has a longer ICU stay, a higher incidence of recurrent nerve injury, and more postoperative morbidity. But we will be able to get our mortality down as low as $3 \%$ with our en bloc group. In terms of postoperative complications, this study has not addressed that.

Dr Mark Krasna (Towson, $M d$ ). Excellent survival results. I have 2 quick questions. The first relates to the group that had transhiatal esophagectomy. Can you tell us, on average, because you had a very good listing of the lymph nodes resected, how many lymph nodes were you able to resect in the transhiatal esophagectomy group?

Dr Lee. When comparing the en bloc versus the non-en bloc, the average is 34 in the en bloc and roughly 17 in the transhiatal group. So we were able to average about 17 lymph nodes.

Dr Krasna. Seventeen for the transhiatal?

Dr Lee. In the transhiatal group. The abdominal dissection is identical. The only difference, obviously, is the mediastinal dissection.
Dr Krasna. And the other question, again, to drill down to something Dr Rice was alluding to, is the use of either neoadjuvant or adjuvant therapy. Because the majority of your failures were distant, I would agree that you definitely want to give these patients chemotherapy. The question would be are you now going to change your regimen and offer all your patients neoadjuvant chemotherapy or are you going to be offering all of your patients adjuvant therapy and, if so, which approach would you choose?

Dr Lee. It is our standard to offer any patients who are stage II or greater disease induction chemotherapy. As we all know, they can tolerate chemotherapy better in the preoperative setting rather than in the postoperative setting.

Dr Raphael Bueno (Boston, Mass). The en bloc technique that you are using, is it still the one in which you take the thoracic duct, sometimes the azygos, and how is that going to be different from the thoracotomy resection? You had a transhiatal and a thoracotomy cohort. How is the thoracotomy cohort different technically from the en bloc technique?

Dr Lee. With a transthoracic non-en bloc, you leave the thoracic duct and then you just take the esophagus. You do not go into the ipsilateral pleura and you do not do a third-field lymph node dissection. So it is the McKeown, and sometimes patients did have the Ivor Lewis, which is a standard non-en bloc resection. 